COMMUNICATIONS IN

ANALYSIS AND GEOMETRY

Volume 11, Number 4, 751-773, 2003

\title{
The Cohomology Rings of Symplectic Quotients
}

\author{
Susan Tolman and Jonathan Weitsman
}

Let $(M, \omega)$ be a symplectic manifold, equipped with a Hamiltonian action of a compact Lie group $G$. We give an explicit formula for the cohomology ring of the symplectic quotient $M / / G$ in terms of the cohomology ring of $M$ and fixed point data. Under certain conditions, our formula also holds for the integral cohomology ring, and can be used to show that the cohomology of the reduced space is torsion-free.

\section{Introduction.}

Let $(M, \omega)$ be a symplectic manifold, equipped with a Hamiltonian action of a compact Lie group $G$. Denote the moment map for the $G$ action by $\phi: M \longrightarrow \mathfrak{g}^{*}=\operatorname{Lie}(G)^{*}$. Assume that 0 is a regular value of $\phi$, and let $M_{\text {red }}:=\phi^{-1}(0) / G$ denote the reduced space. The inclusion $i: \phi^{-1}(0) \longrightarrow M$ induces a map in equivariant cohomology, which, when composed with the natural identification $H_{G}^{*}\left(\phi^{-1}(0)\right) \simeq H^{*}\left(M_{\text {red }}\right)$, yields a map $\kappa: H_{G}^{*}(M) \longrightarrow$ $H^{*}\left(M_{\text {red }}\right)$, called the Kirwan map. According to a theorem of Kirwan, this map is a surjection. The purpose of this paper is to answer the natural question: What is the kernel of $\kappa$ ?

This question was answered explicitly by Kirwan in a number of examples [K1]. In [W] Witten formulated the idea of nonabelian localization: this term refers to a procedure which computes the evaluation on the fundamental class of a symplectic quotient $\int_{M_{\text {red }}} \kappa(\alpha)$ (for any equivariant cohomology class $\alpha$ ) in terms of data on the original manifold $M$. For Witten the data on $M$ consisted of the critical sets of the function $\|\phi\|^{2}$ (the norm squared of the moment map). Motivated by Witten's work, Jeffrey and Kirwan [JK] gave a proof of Witten's results using the geometry of the image of the moment

\footnotetext{
${ }^{1} \mathrm{~S}$. Tolman was partially supported by an NSF Mathematical Sciences Postdoctoral Research Fellowship, by an NSF grant, and by an Alfred P. Sloan Foundation Fellowship. J. Weitsman was supported in part by NSF grant DMS 94/03567, by NSF Young Investigator grant DMS 94/57821, and by an Alfred P. Sloan Foundation Fellowship.
} 
map, and also found an alternative version of nonabelian localization (the residue formula, Section 8 of $[\mathrm{JK}])$ which computes $\int_{M_{\mathrm{red}}} \kappa(\alpha)$ in terms of fixed point data on $M$ (in other words the components of the fixed point set of the maximal torus, the characteristic classes of their normal bundles, the weights of the action of the maximal torus on the normal bundles, and the values of the moment map on the components of the fixed point set) (See also $[\mathrm{GK}, \mathrm{P}, \mathrm{V}])$. Since, by Poincaré duality, $\beta \in H_{G}^{*}(M ; \mathbb{Q})$ is in the kernel of $\kappa$ exactly if the integral of $\kappa(\alpha \beta)$ over $M_{\text {red }}$ is zero for all $\alpha \in H_{G}^{*}(M, \mathbb{Q})$, the kernel of $\kappa$ can, in principle, be computed from their results, in the case of rational cohomology. In practice it is often difficult to compute the ring structure of the cohomology of a manifold in terms of its intersection pairings (or vice versa).

In this paper we give a description of the kernel of $\kappa$ in terms of fixed point data. For according to a theorem of F. Kirwan, the natural restriction map from the equivariant cohomology of $M$ to the equivariant cohomology of the fixed point set is an injection. We compute the kernel of $\kappa$ in terms of the image of this map. This image is well understood in many examples. Moreover, it is determined by the subset of one and zero dimensional orbits (see [GKM, TW] and references therein). Our methods also give a basis for the kernel of $\kappa$, and, under some restrictions, allow us to compute the integral cohomology rings of symplectic quotients.

Let us recall a number of definitions. Let $G$ be a compact Lie group. Choose a maximal torus $T$ and let $W:=N(T) / T$ be the corresponding Weyl group. Let $\epsilon: W \longrightarrow \pm 1$ take the value 1 on all elements of even length, and -1 on all elements of odd length. Choose a positive Weyl chamber, and let $\rho$ denote the product of the positive weights.

Let $E G$ be a contractible space on which $G$ acts freely. Given a $G$ action on a space $X$, define the equivariant cohomology of $X$ as $H_{G}^{*}(X, \mathbb{Q}):=$ $H^{*}\left(X \times_{G} E G, \mathbb{Q}\right)$.

There is a natural fibration $\pi: Y \times_{T} E G \longrightarrow Y \times_{G} E G$, with fiber $G / T$. Moreover, as long as we restrict to rational coefficients, every cohomology class on $G / T$ extends globally to one on $Y \times_{T} E G$. Hence, by the Leray-Hirsch Theorem, there exists an isomorphism between $H_{G}^{*}(X, \mathbb{Q}) \otimes H^{*}(G / T, \mathbb{Q})$ and $H_{T}^{*}(X, \mathbb{Q})$; this isomorphism naturally identifies $H_{G}^{*}(X, \mathbb{Q})$ with the Weyl invariant part of $H_{T}^{*}(X, \mathbb{Q})$, which we shall denote by $H_{T}^{*}(X, \mathbb{Q})^{W}$.

Recall also that the natural projection $X \times_{T} E T \longrightarrow E T / T$ induces a map in cohomology that makes $H_{T}^{*}(X, \mathbb{Q})$ into an $H_{T}^{*}(\cdot, \mathbb{Q})$ module. Additionally, there is a natural identification of $H_{T}^{*}(\cdot, \mathbb{Q})$ with the symmetric algebra in the group of characters of $T$. Composing these two maps, we 
may consider $\rho$ as an element of $H_{T}^{*}(X, \mathbb{Q})$. We can now state the our main theorem.

Theorem 1. Let a compact Lie group $G$ with maximal torus $T$ act on a compact symplectic manifold $M$ with moment map $\phi: M \longrightarrow \mathfrak{g}^{*}$; let $\phi_{T}$ : $M \longrightarrow \mathfrak{t}^{*}$ denote the moment map for the induced action of $T$. Assume that 0 is a regular value of $\phi_{T}$ and of $\phi$. Let $F$ denote the set of fixed points of the $T$ action. For all $\xi \in \mathfrak{t}$, define

$$
\begin{gathered}
M_{\xi}:=\{m \in M \mid\langle\phi(m), \xi\rangle \leq 0\}, \\
K_{\xi}:=\left\{\alpha \in H^{*}(M ; \mathbb{Q})|\alpha|_{F \cap M_{\xi}}=0\right\}, \text { and } \\
K:=\sum_{\xi \in \mathfrak{t}} K_{\xi} .
\end{gathered}
$$

Then, under the identification of $H_{G}^{*}(M, \mathbb{Q})$ with $H_{T}^{*}(M, \mathbb{Q})^{W}$, the kernel of the Kirwan map $\kappa_{G}: H_{T}^{*}(M, \mathbb{Q})^{W} \longrightarrow H^{*}(M / / G, \mathbb{Q})$ is given by

$$
\left\{\rho^{-1} \sum_{w \in W} \epsilon(w) \cdot w(k) \mid k \in K \subset H_{T}^{*}(M, \mathbb{Q})\right\} .
$$

Theorem 1 follows immediately from Proposition 6.1 and Theorem 3 of Section 5. Proposition 6.1 reduces the proof of Theorem 1 to the case of the action of an abelian group; this case is proved in Theorem 3, which is the main technical result of this paper. The main ingredient of the proof of Theorem 3 is an application of Morse theory (as extended by Bott and Kirwan) to functions associated to the moment map $\phi$. Specifically, we study the functions given by the components $\phi^{\xi}$ of the moment map, and by its square $\|\phi\|^{2}$. The Morse theory of these functions has remarkable properties, as shown by Frankel $([\mathrm{F}])$ and by Atiyah and Bott [AB1, AB2]. These properties can be summarized by the statement that as Morse functions, these functions are self-completing, and equivariantly, they are locally selfcompleting. More technically, we choose some critical value $c$ of the relevant function $f$, and suppose that the interval $[c-\epsilon, c+\epsilon]$ contains no critical values of $f$ other than $c$. Write $M_{ \pm}=f^{-1}(-\infty, c \pm \epsilon)$. Then the long exact sequence of the pair $\left(M_{+}, M_{-}\right)$breaks up into short exact sequences

$$
0 \longrightarrow H^{*}\left(M_{+}, M_{-}\right) \longrightarrow H^{*}\left(M_{+}\right) \longrightarrow H^{*}\left(M_{-}\right) \longrightarrow 0,
$$

where the $H^{*}(\cdot)$ denotes either ordinary or equivariant rational cohomology.

When applied to the function $f=\phi^{\xi}$ for some $\xi$ in the Lie algebra of $T$, this sequence can be used to study the equivariant cohomology of $T$. For 
example, Kirwan used this approach to prove that $f$ is a perfect and equivariantly perfect Morse function, and that the cohomology of $M$ is equivariantly formal. More importantly for our purposes, Kirwan also showed that the restriction of the equivariant cohomology of $M$ to the equivariant cohomology of the fixed point set in an injection. This technique is used in a companion paper to analyze the image of this map [TW].

In order to study the symplectic quotient $M_{\text {red }}$, we consider instead the Morse theory associated to the function $f=\|\phi\|^{2}$. This is convenient because the minimum of this function is precisely $\phi^{-1}(0)$, whose equivariant cohomology coincides with the (ordinary) cohomology of $M_{\text {red }}$. In the context of gauge theory such Morse functions were studied by Atiyah and Bott [AB2]; in the context of finite-dimensional manifolds they were studied by Kirwan $[\mathrm{K}]$. This variant of Morse theory is the key ingredient in the proof of the surjectivity theorem cited above. It is also the key element in our proof of Theorem 3 .

The remainder of this paper is structured as follows. In Section 2, we study the key local lemma, due to Atiyah and Bott, which implies the equivariant perfection of the various Morse functions which we will study. We then proceed, in Section 3, to give a simple proof of a version (Theorem 2) of our main theorem which applies to the case where the torus $T$ is onedimensional. This serves to illustrate the basic ideas of our proof in a simple setting, where we may use Morse-Bott theory rather than its elaboration to the case of degenerate Morse functions. We then bite the bullet in Section 4, introducing the ideas of Morse-Kirwan theory that will be required in Section 5, where the main theorem (Theorem 3) is proved for the case of abelian group actions; we include a version for noncompact manifolds as Theorem 4. In Section 6 we show how the formula of Theorem 3 can be combined with the results of Martin (Theorem 6.5 [M], see also Brion [B]) to provide a formula (Theorem 6.1) for the kernel of the Kirwan map in the case of nonabelian group actions. Combined with Theorem 3 , this result gives the main result of our paper (Theorem 1).

The last three sections are devoted to extensions of the basic ideas of the paper. In Section 7, we state and prove versions of our results which hold for cohomology with integer coefficients. In Section 8 we use methods similar to those used in the proof of the main theorem to give a condition for the cohomology rings of symplectic quotients to be torsion-free. Finally in Section 9 we show how our method can be used to compute the cohomology rings of compact smooth projective toric varieties (which are all given as symplectic quotients by tori of Euclidean spaces).

Acknowledgements: We would like to thank Victor Guillemin, who 
encouraged us to consider the consequences for symplectic geometry of the work of Goresky, Kottwitz and MacPherson [GKM]. The present paper and its companion [TW] would not have been possible without his prescient advice. One of us (J.W.) would also like to thank Prof. Guillemin and the MIT Mathematics Department for the opportunity to spend the Spring semester of 1996 at MIT. We would also like to thank R. Sjamaar for pointing out to us the work of Brion [B].

\section{The key lemma.}

The first step in the proof of our main theorem is a local lemma. This lemma, due to Atiyah and Bott, is the key fact behind many of the results we describe.

Lemma 2.1. (Atiyah-Bott) Let a compact Lie group $G$ act on a manifold $X$ and an oriented real vector bundle $E$ over $X$. Assume that a circle subgroup $S^{1} \subset G$ acts on $E$ so that the fixed point set is precisely $X$. Choose an invariant metric on $E$, and let $D$ and $S$ denote the unit disk and sphere bundle, respectively. Then the natural long exact sequence in relative equivariant cohomology splits into a short exact sequence:

$$
0 \longrightarrow H_{G}^{*}(D, S ; \mathbb{Q}) \longrightarrow H_{G}^{*}(D ; \mathbb{Q}) \longrightarrow H_{G}^{*}(S ; \mathbb{Q}) \longrightarrow 0
$$

Since $D$ is homotopic to $X$, we have an identification of $H_{G}^{*}(D ; \mathbb{Q})$ and $H_{G}^{*}(X ; \mathbb{Q})$. Moreover, the Thom isomorphism identifies $H_{G}^{*-\lambda}(X ; \mathbb{Q})$ and $H_{G}^{*}(D, S ; \mathbb{Q})$. Under these identifications, the natural map $H_{G}^{*}(D, S ; \mathbb{Q}) \longrightarrow$ $H_{G}^{*}(D ; \mathbb{Q})$ in the long exact sequence in relative cohomology is identified with the map from $H_{G}^{*-\lambda}(X ; \mathbb{Q})$ to $H_{G}^{*}(X ; \mathbb{Q})$ given by multiplication by the equivariant Euler class of $E$, where $\lambda$ is the dimension of $E$. An alternate formulation of this lemma is that this Euler class is not a zero divisor.

Notice that the corresponding sequence in ordinary cohomology is never exact for any vector bundle of positive rank for dimensional reasons.

\section{The simplest case: $S^{1}$ actions.}

In this section we prove our theorem in a special case. Specifically, we compute the rational cohomology ring of the reduction of a compact symplectic manifold by a circle. The essential features of our argument are already clear in this case. 
Lemma 3.1. Let the circle $S^{1}$ act on a compact symplectic manifold $M$ with a moment map $\phi: M \longrightarrow \mathbb{R}$. Let a function $f: M \longrightarrow \mathbb{R}$ be defined in any of the three following ways: let $f:=\phi^{2}$, let $f:=\phi$, or let $f:=-\phi$.

Let $C$ be a critical set of index $\lambda$ for $f$, and assume that $C$ is the only critical set in $f^{-1}(f(C)-\epsilon, f(C)+\epsilon)$ for $\epsilon>0$. Define $M^{+}:=f^{-1}(-\infty, f(C)+\epsilon)$, and $M^{-}:=f^{-1}(-\infty, f(C)-\epsilon)$. Then there exists a short exact sequence in equivariant cohomology

$$
0 \longrightarrow H_{S^{1}}^{*-\lambda}(C ; \mathbb{Q}) \longrightarrow H_{S^{1}}^{*}\left(M^{+} ; \mathbb{Q}\right) \longrightarrow H_{S^{1}}^{*}\left(M^{-} ; \mathbb{Q}\right) \longrightarrow 0 .
$$

Moreover, the composition of the injection $H_{S^{1}}^{*-\lambda}(C) \longrightarrow H_{S^{1}}^{*}\left(M^{+}\right)$with the restriction map $H_{S^{1}}^{*}\left(M^{+}\right) \longrightarrow H_{S^{1}}^{*}(C)$ is the cup product with the Euler class of the negative normal bundle of $C$.

In other words, every equivariant cohomology class on $M^{-}$extends to $M^{+}$. The restriction to $C$ of cohomology classes on $M^{+}$which vanish on $M^{-}$is injective and its image is the set of multiples of the Euler class of the negative normal bundle of $C$.

Proof. Except in the case where $f=\phi^{2}$ and $C=\phi^{-1}(0)$, the function $f$ is Morse-Bott at every connected component $C$ of the critical set, the set $C$ is a component of the fixed point set, and the negative normal bundle to $C$ is oriented. By Morse-Bott theory and the Thom isomorphism, there is a long exact sequence

$\cdots \longrightarrow H_{S^{1}}^{*-\lambda}(C ; \mathbb{Q}) \longrightarrow H_{S^{1}}^{*}\left(M^{+} ; \mathbb{Q}\right) \longrightarrow H_{S^{1}}^{*}\left(M^{-} ; \mathbb{Q}\right) \longrightarrow H_{S^{1}}^{*+1-\lambda}(C ; \mathbb{Q}) \longrightarrow \cdots$

Moreover, the composition of the map $H_{S^{1}}^{*-\lambda}(C ; \mathbb{Q}) \longrightarrow H_{S^{1}}^{*}\left(M^{+} ; \mathbb{Q}\right)$ with the restriction map from $H_{S^{1}}^{*}\left(M^{+} ; \mathbb{Q}\right) \longrightarrow H_{S^{1}}^{*}(C ; \mathbb{Q})$ is precisely multiplication by the Euler class of the negative normal bundle. Since the circle fixes $C$ but otherwise acts freely on the negative normal bundle, the Euler class is not a zero divisor by Lemma 2.1. Therefore, this composition is injective. Thus the map $H_{S^{1}}^{*-\lambda}(C ; \mathbb{Q}) \longrightarrow H_{S^{1}}^{*}\left(M^{+} ; \mathbb{Q}\right)$ is also injective.

In contrast, the function $\phi^{2}$ is not Morse-Bott at the critical set $C:=$ $\phi^{-1}(0)$. Nevertheless, because $C$ is the minimum of $\phi^{2}$, the spaces $M^{+}$and $C$ are homotopic, and $M^{-}$is empty. So the lemma follows immediately.

Lemma 3.1 leads immediately to the following theorems of Kirwan.

Theorem 3.2. (Kirwan) Let $S^{1}$ act on a compact symplectic manifold $M$ with a moment map and let $F$ denote the fixed point set. The natural restriction $H_{S^{1}}^{*}(M ; \mathbb{Q}) \longrightarrow H_{S^{1}}^{*}(F ; \mathbb{Q})$ is injective. 
This is a corollary of Lemma 3.1 when we take the moment map as our Morse function. It is proved by induction on the proposition that the natural restriction $H_{S^{1}}^{*}\left(\phi^{-1}(-\infty, a) ; \mathbb{Q}\right) \longrightarrow H_{S^{1}}^{*}\left(F \cap \phi^{-1}(-\infty, a) ; \mathbb{Q}\right)$ is an injection for a regular value $a$ with $n$ critical values below it.

Theorem 3.3. (Kirwan) Let $S^{1}$ act on a compact symplectic manifold $M$ with a moment map $\phi: M \longrightarrow \mathbb{R}$. The Kirwan map $\kappa: H_{S^{1}}^{*}(M ; \mathbb{Q}) \longrightarrow$ $H^{*}\left(M_{\text {red }} ; \mathbb{Q}\right)$ is surjective.

This is also a corollary of Lemma 3.1 by an argument analogous to Theorem 3.2 when we take the function $\phi^{2}$ as our Morse function and note that 0 is its minimum value.

We can now state and prove our main theorem for this special case.

Theorem 2. Let $S^{1}$ act on a compact symplectic manifold $M$ with moment map $\phi: M \longrightarrow R$. Assume that 0 is a regular value of $\phi$. Let $F$ denote the set of fixed points; write $M_{-}:=\phi^{-1}(-\infty, 0)$, and $M_{+}:=\phi^{-1}(0, \infty)$. Define

$$
\begin{gathered}
K_{ \pm}:=\left\{\alpha \in H_{S^{1}}^{*}(M ; \mathbb{Q})|\alpha|_{F \cap M_{ \pm}}=0\right\}, \text { and } \\
K:=K_{+} \oplus K_{-} .
\end{gathered}
$$

Then there is a short exact sequence:

$$
0 \longrightarrow K \longrightarrow H_{S^{1}}^{*}(M ; \mathbb{Q}) \stackrel{\kappa}{\longrightarrow} H^{*}\left(M_{\mathrm{red}} ; \mathbb{Q}\right) \longrightarrow 0
$$

where $\kappa: H^{*}(M ; \mathbb{Q}) \longrightarrow H^{*}\left(M_{\text {red }} ; \mathbb{Q}\right)$ is the Kirwan map.

Remark 3.4. More generally, let a circle act on a manifold $M$. A formal moment map is a Morse-Bott function $\Phi: M \longrightarrow \mathbb{R}$ such that the critical points of $\Phi$ correspond exactly to the fixed points. (See Ginzburg-GuilleminKarshon [GGK].) Then, as long as $M$ is compact and 0 is a regular value of $\Phi$, the theorem above is also true for any formal moment map; this follows easily from a quick examination of the proof.

Remark 3.5. Let $i_{ \pm}: M_{ \pm} \longrightarrow M$ denote the natural inclusions. By Theorem $3.2, K_{ \pm}=\operatorname{ker} i_{ \pm}^{*}$. In this case, a simple proof of our theorem can be given by an application of the Mayer-Vietoris sequence to the triple $\left(M, M_{+}, M_{-}\right)$. The proof given below is one which generalizes to actions of higher-rank tori. 
Proof. The injectivity of the inclusion $K \longrightarrow H_{S^{1}}^{*}(M ; \mathbb{Q})$ is obvious, and the surjectivity of the Kirwan map $\kappa: H_{S^{1}}^{*}(M ; \mathbb{Q}) \longrightarrow H^{*}\left(M_{\text {red }} ; \mathbb{Q}\right)$ is Theorem 3.3.

First, we show that $K \subseteq \operatorname{ker}(\kappa)$. By definition, for every $\alpha \in K_{+}$, $\left.\alpha\right|_{F \cap M_{+}}=0$. As in the proof of Theorem 3.2, applying Lemma 3.1 to the function $\phi$ and using induction, we see that $\left.\alpha\right|_{\phi^{-1}(-\infty, \epsilon)}=0$ for small $\epsilon>0$. In particular $\left.\alpha\right|_{\phi^{-1}(0)}=0$. Therefore, $K_{+} \subseteq \operatorname{ker}(\kappa)$. A similar argument using the function $-\phi$ shows that $K_{-} \subseteq \operatorname{ker}(\kappa)$. Therefore, $K=K_{+} \oplus K_{-} \subseteq$ $\operatorname{ker}(\kappa)$.

It remains to show that $\operatorname{ker}(\kappa) \subseteq K$. Order the set of connected components of the fixed point set $\left\{F_{i}\right\}_{i=1}^{N}$ of $M$ so that if $i<j$ then $\phi\left(F_{i}\right)^{2} \leq \phi\left(F_{j}\right)^{2}$. We wish to show that if $\alpha \in H_{S^{1}}^{*}(M ; \mathbb{Q})$ satisfies $\left.\alpha\right|_{\phi^{-1}(0)}=0$, then $\alpha$ is in $K$. By Theorem 4.2 it suffices to find $\alpha^{\prime} \in K$ such that $\left.\alpha\right|_{F_{i}}=\left.\alpha^{\prime}\right|_{F_{i}}$ for all $i$. By induction, it suffices to prove that given $p>0$ and any $\alpha \in H_{S^{1}}^{*}(M ; \mathbb{Q})$ which vanishes on $\phi^{-1}(0)$ and on $F_{i}$ for all $i<p$, there exits $\alpha^{\prime} \in K$ such that $\left.\alpha^{\prime}\right|_{\phi^{-1}(0)}=\left.\alpha\right|_{\phi^{-1}(0)}$ and $\left.\alpha^{\prime}\right|_{F_{i}}=\left.\alpha\right|_{F_{i}}$ for all $i \leq p$.

Assume that we are given such an $\alpha$. Applying Lemma 3.1 to the function $\phi^{2}$, we see that $\left.\alpha\right|_{F_{p}}$ is a multiple of the Euler class $e$ of the negative normal bundle of $F_{p}$ for the function $\phi^{2}$. By symmetry, we may assume that $\phi\left(F_{p}\right)>$ 0 . Then $F_{p}$ is also a critical set for the function $\phi$, and the Euler class of the negative normal bundle of $F_{p}$ for $\phi$ is also $e$. Applying induction and Lemma 3.1 to the function $\phi$, we see that there exists $\alpha^{\prime} \in H_{S^{1}}^{*}(M ; \mathbb{Q})$ such that $\left.\alpha^{\prime}\right|_{F_{p}}=\left.\alpha\right|_{F_{p}}$, and such that the restriction of $\alpha^{\prime}$ to $\phi^{-1}\left(-\infty, f\left(F_{p}\right)-\epsilon\right)$ is trivial for all $\epsilon>0$. In particular, $\left.\alpha^{\prime}\right|_{F_{i}}=0$ for all $i$ such that $\phi\left(F_{i}\right) \leq 0$, and thus $\alpha^{\prime} \in K_{-} \subset K$. Similarly, $\left.\alpha^{\prime}\right|_{F_{i}}=0$ for all $i<p$. The result follows.

\section{Torus actions and Morse-Kirwan theory.}

In this section, we review Morse-Kirwan theory and Kirwan's application of it to the cohomology ring of symplectic manifolds with Hamiltonian torus actions. The basic idea is that in order to generalize the earlier result, we do not really require our function to be a non-degenerate Morse-Bott function. All we need is that our function behave like a Morse function on the level of cohomology, and that Lemma 2.1 holds for the normal bundles of the critical sets. Kirwan shows that although functions arising from the square of the moment map are not non-degenerate, they do satisfy both these properties.

We have already encountered something of this kind in the first section. Let the circle $S^{1}$ act on a compact symplectic manifold $M$ with moment map $\phi: M \longrightarrow \mathbb{R}$. As mentioned in the proof of Lemma 3.1, the critical set 
$\phi^{-1}(0)$ of function $\phi^{2}$ is degenerate. However, as 0 is a minimum, the second Morse lemma still holds at this critical level, so that this kind of degeneracy is inconsequential.

Kirwan developed an extension of Morse theory which applies to functions which have well behaved degeneracies - morally, they look like the product of a minimum and a non-degenerate Morse-Bott function. In particular, she shows that for a critical set $C$ of such a function $f: M \longrightarrow \mathbb{R}$ there is a long exact sequence

$$
\cdots \longrightarrow H_{S^{1}}^{*-\lambda}(C ; \mathbb{Q}) \longrightarrow H_{S^{1}}^{*}\left(M^{+} ; \mathbb{Q}\right) \longrightarrow H_{S^{1}}^{*}\left(M^{-} ; \mathbb{Q}\right) \longrightarrow H_{S^{1}}^{*+1-\lambda}(C ; \mathbb{Q}) \longrightarrow \cdots
$$

and that the composition of the map $H_{S^{1}}^{*-\lambda}(C ; \mathbb{Q}) \longrightarrow H_{S^{1}}^{*}\left(M^{+} ; \mathbb{Q}\right)$ with the restriction map from $H_{S^{1}}^{*}\left(M^{+} ; \mathbb{Q}\right) \longrightarrow H_{S^{1}}^{*}(C ; \mathbb{Q})$ is precisely multiplication by the Euler class of the negative normal bundle to $C$. Here, $M^{+}:=f^{-1}(f(C)+\epsilon)$, and $M^{-}:=f^{-1}(f(C)-\epsilon)$. Kirwan then showed that this extension of Morse theory applies to the square of the moment map for a torus action on a manifold. Finally, she used this theory and Lemma 2.1 to argue exactly as in the proof of Lemma 3.1, and thus prove the following Lemma:

Lemma 4.1. (Kirwan) Let a torus $T$ act on a symplectic manifold $M$ with a proper moment map $\phi: M \longrightarrow \mathfrak{t}^{*}$. Choose a fixed inner product on the dual of the Lie algebra $\mathfrak{t}^{*}$. Let a function be defined $f: M \longrightarrow \mathbb{R}$ in either of the two following ways

1. Given $a \in \mathfrak{t}^{*}$, define $f: M \longrightarrow \mathbb{R}$ by $f(x)=\langle\phi(x)-a, \phi(x)-a\rangle$.

2. Given any $\xi \in \mathfrak{t}$, define $f:=\phi^{\xi}$.

Let $C$ be a critical set of index $\lambda$ for $f$ and assume that $C$ is the only critical set in $f^{-1}(f(C)-\epsilon, f(C)+\epsilon)$ for some $\epsilon>0$. Define $M^{+}:=f^{-1}(-\infty, f(C)+\epsilon)$, and $M^{-}:=f^{-1}(-\infty, f(C)-\epsilon)$. Then there exists a short exact sequence in equivariant cohomology

$$
0 \longrightarrow H_{T}^{*-\lambda}(C ; \mathbb{Q}) \longrightarrow H_{T}^{*}\left(M^{+} ; \mathbb{Q}\right) \longrightarrow H_{T}^{*}\left(M^{-} ; \mathbb{Q}\right) \longrightarrow 0 .
$$

Moreover, the composition of the injection from $H_{T}^{*-\lambda}(C ; \mathbb{Q}) \longrightarrow H_{T}^{*}(M ; \mathbb{Q})$ with the restriction map from $H_{T}^{*}(M ; \mathbb{Q}) \longrightarrow H_{T}^{*}(C ; \mathbb{Q})$ is the cup product with the equivariant Euler class of the negative normal bundle.

The following theorems are direct consequences of this Lemma: 
Theorem 4.2. (Kirwan) Let $T$ act on a symplectic manifold $M$ with a moment map $\phi$. Assume that the set of connected components of $F$, the fixed point set, is finite, and that there exists a generic $\xi \in \mathfrak{t}$ such that $\phi^{\xi}$ is proper and bounded below. Then the natural restriction $H_{T}^{*}(M ; \mathbb{Q}) \longrightarrow H_{T}^{*}(F ; \mathbb{Q})$ is an injection.

This follows by applying Lemma 4.1 to the function $\phi^{\eta}$ for a generic $\eta \in \mathfrak{t}$ close to $\xi$, as in Theorem 3.2.

Note that the restriction map may not be injective if we drop the assumption on the image of $M$; for example, consider the cotangent bundle to $S^{1}$.

Theorem 4.3. (Kirwan) Let $T$ act on a symplectic manifold $M$ with a proper moment map, and assume that the set of connected components of the fixed point set is finite. If 0 is a regular value of $\phi$, then the Kirwan map $\kappa: H_{T}^{*}(M ; \mathbb{Q}) \longrightarrow H^{*}\left(M_{\text {red }} ; \mathbb{Q}\right)$ is a surjection.

This follows by applying Lemma 4.1 to the function $\|\phi\|^{2}$, as in Theorem 3.3 .

\section{The main theorem for abelian group actions.}

We now apply the ideas in the previous section to the case we are interested in: computing the cohomology of reduced spaces.

Theorem 3. Let a torus $T$ act on a compact symplectic manifold $M$ with a moment map $\phi: M \longrightarrow \mathfrak{t}^{*}$. Assume 0 is a regular value of $\phi$. Let $F$ denote the set of fixed points. For all $\xi \in \mathfrak{t}$, define

$$
\begin{gathered}
M_{\xi}:=\{m \in M \mid\langle\phi(m), \xi\rangle \leq 0\}, \\
K_{\xi}:=\left\{\alpha \in H^{*}(M ; \mathbb{Q})|\alpha|_{F \cap M_{\xi}}=0\right\}, \text { and } \\
K:=\sum_{\xi \in \mathfrak{t}} K_{\xi} .
\end{gathered}
$$

Then there is a short exact sequence:

$$
0 \longrightarrow K \longrightarrow H_{T}^{*}(M ; \mathbb{Q}) \stackrel{\kappa}{\longrightarrow} H^{*}\left(M_{\mathrm{red}} ; \mathbb{Q}\right) \longrightarrow 0,
$$

where $\kappa: H_{T}^{*}(M ; \mathbb{Q}) \longrightarrow H^{*}\left(M_{\text {red }} ; \mathbb{Q}\right)$ is the Kirwan map.

Remark 5.1. As before, if we denote by $i_{\xi}$ the inclusion map $i_{\xi}: M_{\xi} \longrightarrow$ $M$, then $K_{\xi}=\operatorname{ker} i_{\xi}^{*}$. 
Proof. The injectivity of the inclusion $K \longrightarrow H_{T}^{*}(M ; \mathbb{Q})$ is obvious. The surjectivity of the Kirwan map $\kappa: H_{T}^{*}(M ; \mathbb{Q}) \longrightarrow H^{*}\left(M_{\text {red }} ; \mathbb{Q}\right)$ is Theorem 4.3. It remains to show that $K=\operatorname{ker}(\kappa)$.

First we show that $K \subseteq \operatorname{ker}(\kappa)$. Choose $\xi \in \mathfrak{t}$ and $\alpha \in K_{\xi}$. By applying Lemma 4.1 to the function $f:=\phi^{\xi}$ inductively, as in the proof of Theorem 4.2 , we get $\left.\alpha\right|_{M_{\xi}}=0$. In particular, $\left.\alpha\right|_{\phi^{-1}(0)}=0$. Since there are only a finite number of distinct $K_{\xi}$ 's, we are done.

It remains to show that $\operatorname{ker}(\kappa) \subset K$. Order the critical sets $\left\{C_{i}\right\}_{i=1}^{N}$ of $\|\phi\|^{2}$ so that $i<j$ exactly if $\left\|\phi\left(C_{i}\right)\right\|^{2}<\left\|\phi\left(C_{j}\right)\right\|^{2}$. (We may assume for simplicity that no two critical sets assume the same value.) We wish to show that if $\alpha \in H_{T}^{*}(M)$ satisfies $\left.\alpha\right|_{\phi^{-1}(0)}=0$, then $\alpha$ is in $K$. By Theorem 4.2 it suffices to find $\alpha^{\prime} \in K$ such that $\left.\alpha\right|_{F}=\left.\alpha^{\prime}\right|_{F}$. By induction, it suffices to prove that given any $p>1$ and any $\alpha \in H_{T}^{*}(M)$ which vanishes on $C_{i}$ for all $i<p$, there exists $\alpha^{\prime} \in K$ such that $\left.\alpha^{\prime}\right|_{C_{i}}=\left.\alpha\right|_{C_{i}}$ for all $i \leq p$. Here, we use the fact that every fixed set is a critical set, and that the first critical set is $C_{1}=\phi^{-1}(0)$.

Assume that we are given such an $\alpha$. Applying Lemma 4.1 to the function $\|\phi\|^{2}$, we see that $\left.\alpha\right|_{C_{p}}$ is a multiple of the Euler class $e$ of the negative normal bundle of $C_{p}$ for the function $\|\phi\|^{2}$. The point $x \in M$ is critical for $\|\phi-a\|^{2}$ exactly if the vector $(\phi(x)-a)_{M}$ is zero, where for any $b \in \mathfrak{t}^{*}, b_{M}$ denotes the vector field on $M$ associated to the one parameter subgroup generated by the element in $\mathfrak{t}$ associated to $b$ by the invariant inner product. Therefore, for any $\lambda \in \mathbb{R}^{+}, C_{p}$ is a critical set for the function $\left\|\phi+\lambda \phi\left(C_{p}\right)\right\|^{2}$. Moreover, the Euler class of the negative normal bundle on $C_{p}$ for this new function is still $e$.

Since $M$ is compact, for sufficiently large $\lambda$, $\left\|\phi(F)+\lambda \phi\left(C_{p}\right)\right\|^{2}<\| \phi\left(C_{p}\right)+$ $\lambda \phi\left(C_{p}\right) \|^{2}$ for all $F \in \mathcal{F}$ whose inner product with $C_{p}$ is negative. Choose such a $\lambda$ and apply Lemma 4.1 to the function $f:=\left\|\phi+\lambda \phi\left(C_{p}\right)\right\|^{2}$. Since $\left.\alpha\right|_{C_{p}}$ is a multiple of $e \in H_{T}^{*}\left(C_{p}\right)$, there exists $\alpha^{\prime} \in H^{*}(M)$ such that $\left.\alpha^{\prime}\right|_{C_{p}}=\left.\alpha\right|_{C_{p}}$ and the restriction $\left.\alpha\right|_{C_{i}}$ is trivial for all $i$ such that $f\left(C_{i}\right)<$ $f\left(C_{p}\right)$. In particular, $\left.\alpha^{\prime}\right|_{F}=0$ for all $C_{i}$ whose inner product with $\phi\left(C_{p}\right)$ is negative. Hence $\alpha^{\prime}$ lies in $K$. Finally, for all $i<p,\left\|\phi\left(C_{i}\right)+\lambda \phi\left(C_{p}\right)\right\|^{2}<$ $\left\|\phi\left(C_{p}\right)+\lambda \phi\left(C_{p}\right)\right\|^{2}$, and hence $\left.\alpha^{\prime}\right|_{C_{i}}=0$.

Remark 5.2. (Chern classes) Theorem 3 allows the cohomology ring of the reduced space $M_{\text {red }}$ to be computed in terms of fixed point data on $M$, namely the cohomology ring of the fixed point set and the restriction of each equivariant cohomology class on $M$ to this set. The Chern classes of the reduced space $M_{\text {red }}$ can also be computed in terms of fixed point data, namely, the restriction of each equivariant Chern class to the fixed point 
set. This is because $\left.T M\right|_{\phi^{-1}(0)}=\pi^{*} T M_{\text {red }} \oplus \mathfrak{t}_{\mathbb{C}}^{*}$, where $\pi: \phi^{-1}(0) \longrightarrow$ $\phi^{-1}(0) / T=M_{\text {red }}$ is the projection, and $\mathfrak{t}_{\mathbb{C}}^{*}$ is a trivial bundle. Thus, in terms of the Kirwan map $\kappa, c_{i}\left(M_{\text {red }}\right)=\kappa\left(c_{i}(M)\right)$. On the other hand, by injectivity, $c_{i}(M)$ is determined by its image in $H_{T}^{*}(F)$.

Remark 5.3. (Computability) The description of the kernel of $\kappa$ given in Theorem 3 is algorithmically computable in the following sense. The cohomology ring $H_{T}^{*}(M)$ is a finitely generated module over $H_{T}^{*}(\mathrm{pt})$. Suppose we are given a finite basis for this module. Then by solving a finite set of linear equations, we can produce a finite basis for the submodule $K_{\xi}$, for any $\xi$. Since only a finite number of elements $\xi \in \mathfrak{t}^{*}$ give rise to distinct submodules $K_{\xi}$, we may produce a finite basis for $K$.

Theorem 4. Let a torus $T$ act on a symplectic manifold $M$ with a moment map $\phi: M \longrightarrow \mathfrak{t}^{*}$. Assume 0 is a regular value. Assume that the set of connected components of the set of fixed points is finite and that there exists $\eta \in \mathfrak{t}$ such that $\phi^{\eta}$ is proper and bounded below. For all $\xi \in \mathfrak{t}$, define

$$
\begin{gathered}
M_{\xi}:=\{m \in M \mid\langle\phi(m), \xi\rangle \leq 0\}, \\
K_{\xi}:=\left\{\alpha \in H^{*}(M ; \mathbb{Q})|\alpha|_{M_{\xi}}=0\right\}, \text { and } \\
K:=\sum_{\xi \in \mathfrak{t}} K_{\xi} .
\end{gathered}
$$

Then there is a short exact sequence:

$$
0 \longrightarrow K \longrightarrow H^{*}(M ; \mathbb{Q}) \stackrel{\kappa}{\longrightarrow} H^{*}\left(M_{\mathrm{red}} ; \mathbb{Q}\right) \longrightarrow 0,
$$

where $\kappa: H_{T}^{*}(M ; \mathbb{Q}) \longrightarrow H^{*}\left(M_{\mathrm{red}} ; \mathbb{Q}\right)$ is the Kirwan map.

The argument in the non-compact case is nearly identical to the proof in the compact case; except that since $\phi^{\xi}$ may not be proper, there may exist cohomology classes which vanish on $M_{\xi} \cap F$ but not on $M_{\xi}$ itself.

\section{Reduction from the non-abelian to the abelian case.}

In this section, we show that, in order to compute the cohomology ring for symplectic quotients by non-abelian groups, it suffices to find the kernel of the Kirwan map for the abelian case.

We begin by repeating some notions from the Introduction. Let $G$ be a compact Lie group. Choose a maximal torus $T$ and let $W:=N(T) / T$ be 
the corresponding Weyl group. Let $\epsilon: W \longrightarrow \pm 1$ take the value 1 on all elements of even length, and -1 on all elements of odd length. Choose a positive Weyl chamber, and let $\rho$ denote the product of the positive weights.

Let $E G$ be any contractible space on which $G$ acts freely. Given a $G$ action on a manifold $X$, define the equivariant cohomology of $X$ to be $H_{G}^{*}(X, \mathbb{Q}):=H^{*}\left(X \times_{G} E G, \mathbb{Q}\right)$.

There is a natural fibration $\pi: Y \times_{T} E G \longrightarrow Y \times_{G} E G$, with fiber $G / T$. Moreover, as long as we restrict to rational coefficients, every cohomology class on $G / T$ extends globally to one on $Y \times_{T} E G$. Hence, by the Leray-Hirsch Theorem, there exists an isomorphism between $H_{G}^{*}(X, \mathbb{Q}) \otimes H^{*}(G / T, \mathbb{Q})$ and $H_{T}^{*}(X, \mathbb{Q})$; this isomorphism naturally identifies $H_{G}^{*}(X, \mathbb{Q})$ with the Weyl invariant part of $H_{T}^{*}(X, \mathbb{Q})$, which we shall denote by $H_{T}^{*}(X, \mathbb{Q})^{W}$.

Recall also that the natural projection $X \times_{T} E T \longrightarrow E T / T$ induces a map in cohomology that makes $H_{T}^{*}(X, \mathbb{Q})$ into an $H_{T}^{*}(\cdot, \mathbb{Q})$ module. Additionally, there is a natural identification of $H_{T}^{*}(\cdot, \mathbb{Q})$ with the symmetric algebra in the group of characters of $T$. Composing these two maps, we may consider $\rho$ as an element of $H_{T}^{*}(X, \mathbb{Q})$. We can now state the main result of this section.

Proposition 6.1. Let a compact Lie group $G$ with maximal torus $T$ act on a compact symplectic manifold $M$ with a $G$ moment map $\phi: M \longrightarrow \mathfrak{g}^{*}$; let $\phi_{T}: M \longrightarrow \mathfrak{t}^{*}$ denote the moment map for the induced action of $T$. Assume that 0 is a regular value of $\phi_{T}$. Let $K$ be the kernel of the Kirwan map for the torus $\kappa_{T}: H_{T}^{*}(M, \mathbb{Q}) \longrightarrow H^{*}(M / / T, \mathbb{Q})$.

Then, under the identification of $H_{G}^{*}(M, \mathbb{Q})$ with $H_{T}^{*}(M, \mathbb{Q})^{W}$, the kernel of the Kirwan map for $G, \kappa_{G}: H_{T}^{*}(M, \mathbb{Q})^{W} \longrightarrow H^{*}(M / / G, \mathbb{Q})$, is given by

$$
\left\{\rho^{-1} \sum_{w \in W} \epsilon(w) \cdot w(k) \mid k \in K \subset H_{T}^{*}(M, \mathbb{Q})\right\} .
$$

Corollary 6.3. Let $L$ be a $W$-submodule of $K$ that generates $K$. Then the kernel of $\kappa_{G}$ is generated by

$$
\left.\left\{\rho^{-1} \sum_{w \in W} \epsilon(w) \cdot w(l \cdot \alpha)\right) \mid l \in L \subset H_{T}^{*}(M, \mathbb{Q}) \text { and } \alpha \in H^{*}(G / T, \mathbb{Q})\right\} .
$$

Moreover, the dimension (over $\mathbb{Q}$ ) of 6.4 is at most that of $L$ itself.

These results were proved in the algebraic case by Brion [B]. The Corollary follows from Proposition 6.1 exactly as in [B]. The main ingredient in 
our extension of the Proposition 6.1 to the symplectic case is the following result of Martin [M].

Theorem 6.5. Let a compact Lie group $G$ with maximal torus $T$ act on a compact symplectic manifold $M$ with a moment map $\phi: M \longrightarrow \mathfrak{g}^{*}$. Let $\phi_{T}: M \longrightarrow \mathfrak{t}^{*}$ be the induced moment map for the restriction of the action to the torus $T$, and assume that 0 is a regular value of $\phi_{T}$. Let $K$ be the kernel of the Kirwan map for the torus $\kappa_{T}: H_{T}^{*}(M, \mathbb{Q}) \longrightarrow H^{*}(M / / T, \mathbb{Q})$. Then the kernel of the natural restriction $H_{T}^{*}(M, \mathbb{Q}) \longrightarrow H_{T}^{*}\left(\phi^{-1}(0), \mathbb{Q}\right)$ is the set of elements

$$
\left\{a \in H_{T}^{*}(M, \mathbb{Q}) \mid a \cdot \rho \in K\right\} .
$$

Proof of Proposition 6.1. By Theorem 6.5 and the discussion above, we see immediately that the kernel for the Kirwan map for $G$ is exactly those $a \in H_{T}^{*}(M, \mathbb{Q})^{W}$ such that $a \cdot \rho$ is in $K$.

We say that $x \in H_{T}^{*}(M, \mathbb{Q})$ is Weyl anti-invariant if $w(x)=\epsilon(w) \cdot x$ for all $w \in W$. Clearly, multiplying any anti-invariant element by $\rho$ will make it anit-invariant. On the other hand, every anti-invariant element is a multiple of $\rho$. Thus, the kernel for the Kirwan map for $G$ is exactly all elements of the form $b / \rho$, where $b \in K$ is Weyl anti-invariant.

Finally, define $p: H_{T}^{*}(M, \mathbb{Q}) \longrightarrow H_{T}^{*}(M, \mathbb{Q})$ by $p(c):=\sum_{w \in W} \epsilon(w) \cdot w(c)$. Clearly $p(\alpha)$ is a surjection to the anti-invariant elements of $H_{T}^{*}(M, \mathbb{Q})$, and it additionally preserves $K$.

Remark 6.6. (Chern classes) As in the abelian case, the Chern classes of the reduced space $M_{\text {red }}$ can be computed in terms of fixed point data, namely, the restriction of each equivariant Chern class to the fixed point set. This is because $T M \cong T\left(\phi^{-1}(0)\right) \oplus \mathfrak{g}^{*}$, while $T(M / / G) \oplus \mathfrak{g} \cong T\left(\phi^{-1}(0)\right)$. Thus $T M \cong T(M / / G) \oplus \mathfrak{g}_{\mathbb{C}}$, and $c(T(M / / G))=\kappa_{G}(c(T M) / \rho)$; and again, by injectivity, $c(T M) \in H_{G}(M, \mathbb{Q})=\left(H_{T}(M, \mathbb{Q})\right)^{W}$ is determined by fixed point data.

Remark 6.7. (Computability) We noted in Remark 5.3 that the description given in Theorem 3 for the kernel of the Kirwan map in the abelian case was effectively computable. The same holds for our description of the kernel of the Kirwan map in the nonabelian case given in in Proposition 6.1. This is because Corollary 6.3 shows that the kernel of $\kappa_{G}$ can be obtained from that of $\kappa_{T}$ by a finite algorithm. 


\section{Extending to the integers.}

So far in this paper we have restricted our attention to rational cohomology. In fact, many of the results we have proved have their analogs in integral cohomology; but there are a number of subtleties which occur.

In order to use any of the methods of this paper, we must verify that an analog of Lemma 2.1 holds over the integers. It is easy to see that the naive generalization of Lemma 2.1 is false. Consider, for example, the trivial plane bundle $\mathbb{C} \oplus \mathbb{C}$ over $\mathbb{R P}^{3}$, and let $S^{1}$ act on the plane bundle by $\lambda \cdot(y, z)=\left(\lambda y, \lambda^{2} z\right)$. Since $S^{1}$ acts trivially on $\mathbb{R P}^{3}$, the equivariant cohomology of $\mathbb{R P}^{3}$ is the tensor product of the cohomology of $\mathbb{R P}^{3}$ and the cohomology of $B S^{1}=\mathbb{C P}^{\infty}$. The Euler class of this bundle is $2 x^{2}$, where $x$ denotes the generator of the cohomology of $\mathbb{C P}^{\infty}$. This class clearly is a zero divisor, as it annihilates the two dimensional cohomology of $\mathbb{R P}^{3}$.

In order to avoid such examples, we have to place some restrictions on the action, or else on the cohomology of the fixed point set. The local result we obtain is the following.

Lemma 7.1. Let a torus $T$ act on an oriented vector bundle $E$ over a manifold $X$, fixing $X$. Assume that there exists $S^{1} \subset T$ which does not fix any point in $E \backslash X$. Assume also that, for each prime $p$, one of the following two conditions is satisfied:

1. The cohomology of $X$ has no $p$ torsion.

2. For every point $m \in E \backslash X$, there exists a $\mathbb{Z} / p \subset T$ which acts freely on $m$.

Choose an invariant metric on $E$, and let $D$ and $S$ denote the unit disk and sphere bundle, respectively. The natural long exact sequence in relative equivariant cohomology splits into a short exact sequence:

$$
0 \longrightarrow H^{*}(D, S ; \mathbb{Z}) \longrightarrow H^{*}(D ; \mathbb{Z}) \longrightarrow H^{*}(S ; \mathbb{Z}) \longrightarrow 0 .
$$

Again, this is equivalent to the statement that the Euler class of $E$ is not a zero divisor. To prove this, it suffices to show that each weight is not a zero divisor. Therefore, it is enough to note that for any $T$-representation on $\mathbb{C}$, the corresponding weight is not divisible by $p$ if there exists a $\mathbb{Z} / p \subset T$ which acts freely on every point except 0 .

Checking the proof of Kirwan's injectivity theorem (Theorem 4.2), it is easy to see that it holds over the integers wherever this version of the Atiyah-Bott lemma holds for every fixed point. Thus: 
Proposition 7.2. Let a torus $T$ act on a symplectic manifold $M$ with a moment map $\phi$. Assume that the set of connected components of $F$, the fixed point set, is finite, and that there exists a generic $\xi \in \mathfrak{t}$ such that $\phi^{\xi}$ is proper and bounded below. Suppose in addition that for every prime $p$, one of the following two conditions is satisfied:

1. The integral cohomology of $F$ has no p-torsion, or:

2. For every point $m \in M$ which is not fixed by the $T$-action, there exists a subgroup of $T$ congruent to $\mathbb{Z} / p$ which acts freely on $m$.

Then the natural restriction $H_{T}^{*}(M ; \mathbb{Z}) \longrightarrow H_{T}^{*}(F ; \mathbb{Z})$ is an injection.

In particular, injectivity holds if the fixed point set has no torsion, or if the action is quasi-free.

Integer analogues of Kirwan's surjectivity theorem (Theorem 4.3), and our main result (Theorem 3) also hold under a similar set of restrictions in the case of an $S^{1}$ action. Once again, the proof amounts to noting that the proofs given earlier go through without change as long as the Atiyah-Bott lemma holds for the negative normal bundle at each critical point. In this case, each critical point is either fixed, in which case we apply Lemma 7.1 above, or the minimum, which works automatically.

Proposition 7.3. Let $S^{1}$ act on a compact symplectic manifold $M$ with a moment map $\phi: M \longrightarrow \mathbb{R}$. Assume that for every prime $p$, one of the following two conditions is satisfied:

1. The integral cohomology of $F$ has no p-torsion, or:

2. For every point $m \in M$ which is not fixed by the $T$-action, there exists a subgroup of $T$ congruent to $\mathbb{Z} / p$ which acts freely on $m$.

Then the Kirwan map $\kappa: H_{S^{1}}^{*}(M ; \mathbb{Z}) \longrightarrow H^{*}\left(M_{\text {red }} ; \mathbb{Z}\right)$ is surjective.

Likewise,

Proposition 7.4. Let $S^{1}$ act on a compact symplectic manifold $M$ with moment map $\phi: M \longrightarrow R$. Assume that 0 is a regular value. Let $F$ denote the set of fixed points. Assume that for every prime $p$, one of the following two conditions is satisfied:

1. The integral cohomology of $F$ has no p-torsion, or:

2. For every point $m \in M$ which is not fixed by the $T$-action, there exists a subgroup of $T$ congruent to $\mathbb{Z} / p$ which acts freely on $m$. 
Define

$$
\begin{gathered}
K_{+}:=\left\{\alpha \in H_{S^{1}}^{*}(M ; \mathbb{Z})|\alpha|_{F_{+}}=0\right\}, \text { where } F_{+}:=F \cap \phi^{-1}(0, \infty) ; \\
K_{-}:=\left\{\alpha \in H_{S^{1}}^{*}(M ; \mathbb{Z})|\alpha|_{F_{-}}=0\right\}, \text { where } F_{-}:=F \cap \phi^{-1}(-\infty, 0) ; \text { and } \\
K:=K_{+} \oplus K_{-} .
\end{gathered}
$$

Then there is a short exact sequence:

$$
0 \longrightarrow K \longrightarrow H_{S^{1}}^{*}(M ; \mathbb{Z}) \stackrel{\kappa}{\longrightarrow} H^{*}\left(M_{\mathrm{red}} ; \mathbb{Z}\right) \longrightarrow 0,
$$

where $\kappa: H^{*}(M ; \mathbb{Z}) \longrightarrow H^{*}\left(M_{\text {red }} ; \mathbb{Z}\right)$ is the Kirwan map.

Remark 7.5. In the case where $S^{1}$ does not act freely on $\phi^{-1}(0)$, the cohomology group $H^{*}\left(M_{\text {red }}, \mathbb{Z}\right)$ which enters into both these results is the orbifold cohomology of $M_{\text {red }}$, not the cohomology of the underlying topological space. These are of course identical over $\mathbb{R}$ but are, in general, different over $\mathbb{Z}$.

On the other hand, when a higher-rank torus $T$ acts on a manifold, Kirwan's surjectivity Theorem 4.3, and our main result (Theorem 3) require a more subtle application of the Atiyah-Bott Lemma, in that the critical points of the Morse function $\phi^{2}$ include spaces more complicated than the fixed points. In order to generalize our proofs, we need a version of Lemma 7.1 which holds at all of these critical points. There are various conditions under which this can be seen to hold, but the statements of these conditions can be cumbersome. We therefore restrict our attention to the quasi-free case, where the Euler class of the negative normal bundle (at any critical manifold) is not a zero divisor; thus the conditions of Lemma 6.1 are met at every point of the fixed set.

Proposition 7.6. (Kirwan) Let $T$ act quasi-freely on a symplectic manifold $M$ with a proper moment map, and assume that the set of connected components of the fixed point set is finite.

If 0 is a regular value, then the Kirwan map $\kappa: H_{T}^{*}(M ; \mathbb{Z}) \longrightarrow$ $H^{*}\left(M_{\mathrm{red}} ; \mathbb{Z}\right)$ is a surjection.

Finally we have an integer version of our main result:

Proposition 7.7. Let a torus $T$ act quasi-freely on a symplectic manifold $M$ with a moment map $\phi: M \longrightarrow \mathbb{R}$. Assume 0 is a regular value. Assume that the set of connected components of $F$, the set of fixed points, is finite and that there exists $\xi \in \mathfrak{t}$ such that $\phi^{\xi}$ is proper and bounded below. For 
all $\xi \in \mathfrak{t}$, define $K_{\xi}$ to be the set of $\alpha \in H^{*}(M ; \mathbb{Z})$ such that $\alpha$ vanish when restricted to the set $M_{\xi}=\left(\phi^{\xi}\right)^{-1}(0, \infty)$. Define $K:=\sum_{\xi \in \mathfrak{t}} K_{\xi}$. Then there is a short exact sequence:

$$
0 \longrightarrow K \longrightarrow H^{*}(M ; \mathbb{Z}) \stackrel{\kappa}{\longrightarrow} H^{*}\left(M_{\mathrm{red}} ; \mathbb{Z}\right) \longrightarrow 0,
$$

where $\kappa: H_{T}^{*}(M ; \mathbb{Z}) \longrightarrow H^{*}\left(M_{\text {red }} ; \mathbb{Z}\right)$ is the Kirwan map.

\section{Torsion-free reduced spaces.}

In a large class of examples, the integer cohomology of the reduced spaces is torsion-free. We begin with the case of reduction by an $S^{1}$ action.

Theorem 5. Let the circle $S^{1}$ act on a compact symplectic manifold $M$ with a moment map $\phi$. Assume that the integer cohomology $H^{*}(F ; \mathbb{Z})$ of the fixed point set $F$ is torsion-free, and that $S^{1}$ acts freely on $\phi^{-1}(0)$. Then the integer cohomology ring of the reduced space is also torsion-free.

Proof. Note first that because the fixed point set is torsion-free, the conditions of Propositions 6.2 and 6.3 are met. Assume that $p \in \mathbb{Z}$ is a prime and $\alpha \in H_{S^{1}}^{*}(M ; \mathbb{Z})$ is a cohomology class and $\left.p \alpha\right|_{\phi^{-1}(0)}=0$. We must show that $\left.\alpha\right|_{\phi^{-1}(0)}=0$. Order the set of connected components of the fixed point set $\left\{F_{i}\right\}_{i=1}^{N}$ of $M$ so that if $i<j$ then $\phi\left(F_{i}\right)^{2} \leq \phi\left(F_{j}\right)^{2}$. By Theorem 4.2 it suffices to find $\alpha^{\prime} \in H_{S^{1}}^{*}(M ; \mathbb{Z})$ such that $\left.\alpha^{\prime}\right|_{\phi^{-1}(0)}=0$ and $\alpha_{F_{i}}=\left.\alpha^{\prime}\right|_{F_{i}}$ for all $i$. By induction, it suffices to prove that given an integer $q>0$ and any $\alpha \in H_{S^{1}}^{*}(M ; \mathbb{Z})$ which vanishes on $F_{i}$ for all $i<q$ and such that $p \alpha$ vanishes on $\phi^{-1}(0)$, there exists $\alpha^{\prime} \in H_{S^{1}}^{*}(M ; \mathbb{Z})$ such that and $\left.\alpha^{\prime}\right|_{F_{i}}=\left.\alpha\right|_{F_{i}}$ for all $i \leq q$ and $\alpha^{\prime}$ vanishes on $\phi^{-1}(0)$.

Assume that we are given such an $\alpha$. Applying Lemma 3.1 to the function $\phi^{2}$ and the class $p \alpha$, we see that $\left.p \alpha\right|_{F_{q}}$ is a multiple of the Euler class $e$ of the negative normal bundle of $F_{q}$ for the function $\phi^{2}$. We now have two cases two consider.

First, assume that $p \nmid e$. Then, by Lemma 8.1, $\left.\alpha\right|_{F_{q}}$ is also a multiple of $e$. By symmetry, we may assume that $\phi\left(F_{q}\right)>0$. Then $F_{q}$ is also a critical set for the function $\phi$, and the Euler class of the negative normal bundle of $F_{q}$ for $\phi$ is still $e$. Applying induction and Lemma 3.1 to the function $\phi$, we see that there exists $\alpha^{\prime} \in H_{S^{1}}^{*}(M ; \mathbb{Q})$ such that $\left.\alpha^{\prime}\right|_{F_{q}}=\left.\alpha\right|_{F_{q}}$, and such that the restriction of $\alpha^{\prime}$ to $\phi^{-1}\left(-\infty, f\left(F_{q}\right)-\epsilon\right)$ is trivial for all $\epsilon>0$. In particular, $\left.\alpha^{\prime}\right|_{F_{i}}=0$ for all $i<p$. and $\alpha^{\prime}$ vanishes on $\phi^{-1}(0)$. 
More generally, let $N \subset M$ be the connected component of the set of points which is fixed by $\mathbb{Z} /(p)$ which contains $F_{q}$. Let $a$ be the downward Euler class for $F_{q}$ in $N$ using the Morse-Bott function $\phi$, an $b$ be the Euler class for the complimentary bundle, so $e=a b$. Since the action on $\phi^{-1}(0)$ is free, the induction hypothesis assures that $\left.\alpha\right|_{F_{i}}=0$ for all $i$ such that $\phi\left(F_{i}\right)<\phi\left(F_{q}\right)$ and $F_{i} \subset N$ Therefore $\left.\alpha\right|_{F_{q}}=a \eta$, for some $\eta$. Since $\left.p \alpha\right|_{F_{q}}=$ $e \xi=a b \xi$, we can substitute to get $p a \eta=a b \xi$. Since $a$ cannot be a zerodivisor, this implies that $p \eta=b \xi$. As before, this implies that $\xi=p \xi^{\prime}$, etc.

Lemma 8.1. Let a compact torus $T$ act on an oriented real vector bundle $E$ over a manifold $X$. Assume that a circle subgroup $S^{1} \subset T$ acts on $E$ so that the fixed point set of $S^{1}$ and $\mathbb{Z} /(p) \subset S^{1}$ is precisely $X$ for $p \in \mathbb{N}$. Assume also that $H^{*}(X ; Z)$ is torsion-free. Let e be the equivariant Euler class of $E$. Then for any cohomology classes $\alpha$ and $\beta$ such that $p \alpha=\xi e$, there exists $\xi^{\prime}$ such that $p \xi^{\prime}=\xi$.

We start by assuming that $X$ is fixed by $T$.

Proof. Identify the torus $T$ with $\left(S^{1}\right)^{n}$. Order $n$-tuples as follows: first by the sum of all coordinates, then by the first coordinate, the second coordinate, etc. Because the action fixes $X$, the cohomology ring $H_{T}^{*}(X ; \mathbb{Z})$ is simply the polynomial ring in $n$ generators on $H^{*}(X ; \mathbb{Z})$. Every term of $e$ such that the sum of the coefficients is $\lambda$ is an integer, where $\lambda$ is the rank of $E$. Because the action is $p$-free outside $X, p$ cannot divide all these integers. Let $I$ be the largest $n$-tuple such that $p \not \xi_{I}$, and $J$ be the largest $n$-tuple such that $p \nmid e_{J}$. Note that be the above discussion $e_{J}$ is an integer. Now consider the coefficients of the term $X^{I+J}$ in the expression $p \alpha=e \xi$. It is

$$
\alpha_{I+J}=\sum_{I^{\prime}+J^{\prime}=I+J} e_{I^{\prime}} \xi_{J^{\prime}}
$$

But if $I^{\prime}+J^{\prime}=I+J$, then either $I=I^{\prime}$ and $J=J^{\prime}$ or $I^{\prime}>I$ or $J^{\prime}>J$. Therefore, $e_{I} \xi_{J}=p \beta$, for some $\beta$. We can find integers $s$ and $t$ so that $1=s p+t e_{I}$. Therefore, $\xi_{J}=s p \xi_{J}+t e_{I} \xi_{J}=p\left(s \xi_{J}+t \beta\right)$.

Remark 8.2. Note that if $S^{1}$ does not act freely on $\phi^{-1}(0)$, then the the cohomology of the reduced space must have torsion.

By examining the proof of Theorem 5, we see what difficulties will arise in the case of reduction by the action of a torus of rank $r>1$ : we require 
surjectivity of the Kirwan map, which, in turn, requires a condition on either the stabilizers of points or on the cohomology of critical points of the square of the moment map. These are themselves reduced spaces, whose cohomology being torsion-free will depend on a version of Theorem 5 for reduction by tori of rank $r-1$. Thus the reduced spaces will be torsion-free under a complicated set of conditions, defined inductively. These conditions can easily be verified in examples but are not so easy to state in full generality; we content ourselves with the following statement.

Theorem 6. Let a torus $T$ act quasi-freely on a symplectic manifold $M$ with moment map $\phi$. Suppose 0 is a regular value of the moment map, and that the integer cohomology $H^{*}(F ; \mathbb{Z})$ of the fixed point set $F$ is torsion-free. Then the cohomology $H^{*}\left(M_{\text {red }} ; \mathbb{Z}\right)$ of the reduced space $M_{\text {red }}$ is also torsion-free.

\section{Example: Smooth Toric Varieties.}

In this section, to demonstrate our theorem, we show how it provides a simple method to obtain the cohomology rings of smooth compact projective toric varieties (see e.g. [D]). These compact symplectic manifolds can all be obtained as symplectic quotients of Euclidean spaces.

The moment map $\psi: \mathbb{C}^{N} \longrightarrow \mathbb{R}^{N^{*}}$ for the natural action of $\left(S^{1}\right)^{N}$ on $\mathbb{C}^{N}$ is $\psi\left(z_{1}, \ldots, z_{N}\right)=\left(\left|z_{1}\right|^{2}, \ldots,\left|z_{N}\right|^{2}\right)$. The image of this moment map is given by $\psi\left(\mathbb{C}^{N}\right)=\left\{\xi \in \mathbb{R}^{N^{*}} \mid \xi_{i} \geq 0\right.$ for all $\left.i\right\}$.

Consider a subgroup $G \subset\left(S^{1}\right)^{N}$. This inclusion gives rise to a short exact sequence of Lie algebras

$$
0 \longrightarrow \mathfrak{g} \stackrel{i}{\longrightarrow} \mathbb{R}^{N} \stackrel{\pi}{\longrightarrow} \mathfrak{t} \longrightarrow 0
$$

and its dual sequence

$$
0 \longrightarrow \mathfrak{t}^{*} \stackrel{\pi^{*}}{\longrightarrow} \mathbb{R}^{N^{*}} \stackrel{i^{*}}{\longrightarrow} \mathfrak{g}^{*} \longrightarrow 0
$$

For any $\eta \in \mathfrak{g}^{*}$, the function $\phi:=i^{*} \circ \psi-\eta$ is a moment map for the induced action of $G$ on $\mathbb{C}^{N}$. Assume that $\phi$ is proper, and that 0 is a regular value of $\phi$. Let $M$ be the reduction of $\mathbb{C}^{N}$ by $G$ at 0 . The torus $T:=\left(S^{1}\right)^{N} / G$ acts symplectically on $M$. The image of moment map for the $T$ action can be identified with the polytope $\Delta:=\left\{\xi \in \mathbb{R}^{N^{*}} \mid \xi_{j} \geq\right.$ 0 for all $j$ and $\left.i^{*}(\xi)-\eta=0\right\}$.

There is a (possibly empty) facet of $\Delta$ for each $i \in(1, \ldots, N)$, given by $\xi \in \mathbb{R}^{N^{*}}$ such that $\xi_{j} \geq 0$ for all $j, i^{*}(\xi)-\eta=0$ and $\xi_{i}=0$. For 
$I \subset(1, \ldots, N)$, we say that the $I$ facets intersect if the intersection of these sets is not empty.

Since $\operatorname{dim} T=\frac{1}{2} \operatorname{dim} M, M$ is a compact smooth toric variety.

Theorem 7. Let the ideals $\mathcal{I}$ and $\mathcal{J} \subset \mathbb{Q}\left[x_{1}, \ldots, x_{N}\right]$ be defined as follows: $\mathcal{J}=\left\{\sum \alpha_{i} x_{i} \mid \alpha \in \pi^{*}\left(\mathfrak{t}^{*}\right)\right\}$, and $\mathcal{I}$ is the ideal generated by $\prod_{i \in I} x_{i}$ for all $I \subset(1, \ldots, N)$ such that the $I$ facets do not intersect in the polytope $\Delta$. The cohomology ring $H^{*}(M ; \mathbb{Q})$ is given by $\mathbb{Q}\left[x_{1}, \ldots, x_{N}\right] /(\mathcal{I}, \mathcal{J})$.

Proof. The $G$-equivariant cohomology of $\mathbb{C}^{N}$ can be expressed as

$$
H_{G}^{*}\left(\mathbb{C}^{N} ; \mathbb{Q}\right)=\mathbb{Q}\left[x_{1}, \ldots, x_{N}\right] / \mathcal{J} .
$$

From the construction of $\phi$, it is clear that since $\phi$ is proper, there exists $\zeta$ such that $\phi^{\zeta}$ is proper and bounded below. Therefore, we may apply Theorem 4.

Consider a generic $\xi \in \mathfrak{g}$. The only critical point of $\phi^{\xi}$ is 0 , and the only critical value is $-\langle\eta, \xi\rangle$. If $\langle\eta, \xi\rangle>0$, then $0 \in M_{\xi}$. Since every cohomology class which vanishes when restricted to 0 is trivial, $K_{\xi}=0$.

Now consider the case $\langle\eta, \xi\rangle<0$. The subbundle of the tangent space at 0 consisting of points with all the coordinates zero except the $i$ 'th is in the negative normal bundle for $\phi^{\xi}$ exactly if $\left\langle\beta_{i}, \xi\right\rangle<0$, where $\beta_{i}=\pi^{*}\left(e_{i}\right)$, and the $e_{i}$ 's are the standard basis of $\mathbb{R}^{N^{*}}$. Therefore, $K_{\xi}$ is generated by $\prod_{i \in I} x_{i}$, where $I:=\left\{i \in(1, \ldots, N) \mid\left\langle\beta_{i}, \xi\right\rangle<0\right\}$. (We know that its restriction to 0 must be of this form, and every class is determined by its restriction to 0 .)

Putting this together, it is clear that $K$ is generated by the products $\prod_{i \in I} x_{i}$, over all sets $I$ such that there exists $\xi$ with $\langle\eta, \xi\rangle<0$ and $\left\langle\beta_{j}, \xi\right\rangle>0$ for all $j \notin I$.

Given $I \subset(1, \ldots, N)$ it is clear that $\eta$ is not in $\sum_{j \notin I} \mathbb{R}^{+} \beta_{j}$ exactly if there exists $\xi$ such that $\langle\eta, \xi\rangle<0$ and $\left\langle\beta_{j}, \xi\right\rangle>0$ for all $j \notin I$. Thus, $K$ is generated by $\prod_{i \in I} x_{i}$ for all sets $I$ such that $\eta$ is not in $\sum_{j \notin I} \mathbb{R}^{+} \beta_{j}$. Finally,

$\eta$ is in $\sum_{j \notin I} \mathbb{R}^{+} \beta_{j}$ exactly if the facets corresponding to the elements of $I$ intersect in $\Delta$.

\section{References.}

[A] M. Audin, The Topology of Torus Actions on Symplectic Manifolds. Progress in Mathematics 93, Birkhauser, Boston (1991).

[AB1] M. Atiyah and R. Bott, The moment map and equivariant cohomology, Topology 23 (1984) 1-28. 
[AB2] M.F. Atiyah and R. Bott, The Yang-Mills equations over Riemann surfaces, Phil. Trans. Roy. Soc. Lond. A308 (1982) 523-615.

[B] M. Brion, Cohomologie equivariante des points semi-stables, J. Reine Angew. Math. 421, 125-140 (1991)

[D] V. I. Danilov, The geometry of toric varieties, Russian Math. Surveys, 33, 97-154 (1978).

[GGK] V. Ginzburg, V. Guillemin, and Y. Karshon. Cobordism theory and localization formulas for Hamiltonian group actions, Int. Math. Res. Notices 5 (1996), 221-234.

[GKM] M. Goresky, R. Kottwitz, and R. MacPherson. Equivariant cohomology, Koszul duality, and the localization theorem, Inv. Math., to appear.

[GK] V. Guillemin, and J. Kalkman, The Jeffrey-Kirwan localization theorem and residue operations in equivariant cohomology, J. Reine. Angew. Math. 470, 123-142 (1996)

[F] T. Frankel, Fixed points on Kähler manifolds, Ann. Math. 70, 1-8 (1959).

[JK] L.C. Jeffrey and F.C. Kirwan, Localization for nonabelian group actions. Topology 34, 291-327 (1995).

[K] F. C. Kirwan, The cohomology of quotients in symplectic and algebraic geometry, Princeton University Press, 1984.

[K1] F. C. Kirwan, The cohomology rings of moduli spaces of vector bundles over Riemann surfaces, J. Amer. Math. Soc., 5, 853-906 (1992)

[M] S. K. Martin, personal communication.

[P] P. Paradan, Formule de localisation en cohomologie equivariante. ENS Thesis, 1996.

[TW] S. Tolman and J. Weitsman, On the cohomology rings of Hamiltonian T-spaces, preprint.

[V] M. Vergne, A note on the Jeffrey-Kirwan-Witten localization formula, Topology 35, 243-266 (1996) 
[W] E. Witten, Two dimensional gauge theories revisited, preprint hepth/9204083; J. Geom. Phys. 9 (1992) 303-368.

Susan TOlman

Department of Mathematics

UNIVERSITY OF ILLINOIS AT URBANA-ChAMPAIGN

URBANA, IL 61801

stolman@math.uiuc.edu

JONATHAN WEITSMAN

Department of Mathematics

UNIVERSITY OF CALIFORNIA

Santa Cruz, CA 95064

weitsman@cats.ucsc.edu

ReCEIVED April 21, 2000. 